\title{
Pulmonary microRNA profiling: implications in upper lobe predominant lung disease
}

David A. Armstrong ${ }^{1 *}$, Amanda B. Nymon', Carol S. Ringelberg², Corina Lesseur ${ }^{3}$, Haley F. Hazlett², Louisa Howard², Carmen J. Marsit ${ }^{4}$ and Alix Ashare ${ }^{1,2}$

\begin{abstract}
Background: Numerous pulmonary diseases manifest with upper lobe predominance including cystic fibrosis, smoking-related chronic obstructive pulmonary disease, and tuberculosis. Zonal hypoxia, characteristic of these pulmonary maladies, and oxygen stress in general is known to exert profound effects on various important aspects of cell biology. Lung macrophages are major participants in the pulmonary innate immune response and regional differences in macrophage responsiveness to hypoxia may contribute in the development of lung disease. MicroRNAs are ubiquitous regulators of human biology and emerging evidence indicates altered microRNA expression modulates respiratory disease processes. The objective of this study is to gain insight into the epigenetic and cellular mechanisms influencing regional differences in lung disease by investigating effect of hypoxia on regional microRNA expression in the lung.

All studies were performed using primary alveolar macrophages $(n=10)$ or bronchoalveolar lavage fluid $(n=16)$ isolated from human subjects. MicroRNA was assayed via the NanoString nCounter microRNA assay.

Results: Divergent molecular patterns of microRNA expression were observed in alternate lung lobes, specifically noted was disparate expression of miR-93 and miR-4454 in alveolar macrophages along with altered expression of miR-451a and miR-663a in bronchoalveolar lavage fluid. Gene ontology was used to identify potential downstream targets of divergent microRNAs. Targets include cytokines and matrix metalloproteinases, molecules that could have a significant impact on pulmonary inflammation and fibrosis.
\end{abstract}

Conclusions: Our findings show variant regional microRNA expression associated with hypoxia in alveolar macrophages and BAL fluid in the lung-upper vs lower lobe. Future studies should address whether these specific microRNAs may act intracellularly, in a paracrine/endocrine manner to direct the innate immune response or may ultimately be involved in pulmonary host-to-pathogen trans-kingdom cross-talk.

Keywords: MicroRNA, Epigenetics, Hypoxia, Alveolar macrophages, Exosomes, Microvesicles, Bronchoalveolar lavage

\section{Background}

Numerous pulmonary diseases occur with upper lobe predominance including cystic fibrosis (CF), smokingrelated chronic obstructive pulmonary disorder (COPD), tuberculosis, and sarcoidosis [1]; however, the initiating molecular events of the regional inflammatory response in these diseases have yet to be determined.

In the healthy lung, a physiological hypoxic oxygen gradient exists from the apex $\left(21 \% \mathrm{O}_{2}\right)$ to the base $\left(10-11 \% \mathrm{O}_{2}\right)$ of the lung in the upright position $[2,3]$.

\footnotetext{
* Correspondence: David.A.Armstrong@hitchcock.org

'Pulmonary and Critical Care Medicine, Dartmouth-Hitchcock Medical Center, Lebanon, $\mathrm{NH}$, USA

Full list of author information is available at the end of the article
}

Physiological factors such as lymphatic flow, as well as metabolic factors such as uptake of $\mathrm{O}_{2}$, elimination of $\mathrm{CO}_{2}$, and $\mathrm{pH}$ of airway surface liquid, differ regionally within the lung due to inequalities of the ventilationperfusion ratio [1]. Hypoxia is known to exert profound effects on various important aspects of cell biology, for example, expression of cell surface markers, cell viability, phagocytosis, metabolic activity, and cytokine release $[4,5]$.

Alveolar macrophages (AM) are immune cells that play a pivotal role in the detection and elimination of pathogenic microorganisms [6]. AMs are one of multiple cell types, including type I and type II epithelial cells, endothelial cells and fibroblasts that reside within the 
lung alveoli [7]. Macrophages are phagocytic cells and crucial effectors of innate immunity in the primary response to pathogens besides their key role in acute and chronic inflammatory responses. Many pathological processes with macrophage involvement (e.g., inflammation, wound healing, atherosclerosis, and tumors) are characterized by hypoxia. As macrophages are of paramount importance in the first line defense against invasive microorganisms, it has long been hypothesized that these cells must be especially equipped to cope with and function in hypoxic areas [4].

Epigenetics is the study of heritable changes in gene function caused by mechanisms other than changes in the underlying DNA sequence [8]. One important mode of epigenetic regulation is expression of microRNAs (miRNA) [9]. MicroRNAs are ubiquitous regulators of human biology [10] and increasingly being recognized as important modulators in respiratory disease [11, 12]. MicroRNAs are a class of non-coding RNAs of 19 to 24 nucleotides in length that regulate gene expression through post-transcriptional, RNA interference, and gene silencing pathways. Initially thought to act primarily intracellularly, circulating miRNAs have gained attention as extracellular messengers and have been found associated with actively secreted exosomes/microvesicles and HDL particles $[13,14]$. The number of annotated human microRNA loci currently numbers at more than 2500 in the latest version of miRBase (v.21) [15]. Functionally, in the context of cell-to-cell communication in disease, by working to fine-tune protein expression levels, miRNAs can contribute to regulatory circuits by providing quantitative control of gene output. In particular, miRNAs potentially exert their influence by regulating dosage-sensitive genes for which small fluctuations in protein expression may contribute to a substantial functional output [16]. In addition to their role in host cell-to-cell communication, microRNAs have been implemented in an emerging field of study in higher eukaryotes referred to as trans-kingdom cross-talk, whereby RNA-signal exchange have been described to occur between organisms of different kingdoms [17]. This topic is currently underexplored in pulmonary disease.

Regional differences in resident lung macrophage and other cells' responsiveness to hypoxia may be an important factor in the initial development of pulmonary diseases with upper lobe predominance. Hypoxia has been implicated as an important proximal regulator of miRNA biogenesis and function [18]. Furthermore, as miRNAs have emerged as important epigenetic regulators of the innate and adaptive immune response [19], we assessed microRNA content of upper lobe and lower lobe lung macrophages based on conditional hypoxia as well as upper lobe and lower lobe bronchoalveolar lavage (BAL) fluid for altered microRNA expression.
The objective of this study is to gain insight into the cellular mechanisms influencing pulmonary diseases occurring with upper lobe predominance, specifically understanding how hypoxia may impact the regional innate immune response and if microRNAs may in part be responsible.

\section{Methods}

This study was approved by the Committee for the Protection of Human Subjects at the Geisel School of Medicine at Dartmouth (\#22781). Following written informed consent, subjects underwent flexible bronchoscopy to obtain BAL fluid and primary alveolar macrophages (AM). This procedure has been previously described in detail [20]. Primary AM were isolated from BAL fluid via gravity filtration through 2 layers of gauze, followed by three sequential washes in normal saline with subsequent centrifugation to pellet cells $(400 \times \mathrm{g} / 5 \mathrm{~min} /$ $20{ }^{\circ} \mathrm{C}$ ). AM were resuspended in RPMI 1640 media (50 $\mu \mathrm{g} / \mathrm{ml}$ glutamax/gentamicin) and equilibrated overnight in vitro (normoxic) and then subject to continued normoxic $\left(21 \% \mathrm{O}_{2}\right)$ or hypoxic conditions $\left(5 \% \mathrm{O}_{2}\right)$. Total RNA was extracted from AM using Zymo Quick-RNA Mini-prep Kit (Irvine, CA). Cell-free microRNA from BAL fluid was obtained using Norgen Urine Exosome RNA Isolation Kit (Norgen Biotek Corp., Thorold, ON, Canada) from a $0.3 \mathrm{ml}$ volume of cell-free BAL fluid. All protocols were performed according to manufacturer's instructions. The digital multiplexed NanoString nCounter human v3 microRNA expression assay (NanoString Technologies, Seattle, WA) was performed according to manufacturer's instructions with total RNA or miRNAs extracted as above. Briefly, 3-10 ng microRNA (BAL fluid) or $100 \mathrm{ng}$ total RNA (AM) samples were prepared by ligating a specific miR-tag onto the 3 ' end of each mature miRNA followed by an overnight hybridization $\left(65^{\circ} \mathrm{C}\right)$ to nCounter Reporter and Capture probes. Excess Reporter and Capture probes are washed away using the automated nCounter sample prep station and probe/target complexes are aligned and immobilized in the nCounter Cartridge. Cartridges are then placed in the nCounter digital analyzer for data collection. nSolver Analysis software (NanoString) (V3.0) was used for data analysis including background correction by subtracting the mean of the six negative controls included on the NanoString platform and normalization using the average geometric mean of the top 100 probes detected. Additional methods including: total RNA/microRNA extraction/purification/quantification, droplet digital PCR, ELISAs, and electron microscopy are detailed in the on-line data supplement-methods section (Additional file 1). Bio-analyzer electropherograms for microRNA quantitation are presented in on-line Additional file 2: Figure S1 and NanoString technical replicates are show in on-line Additional file 3: Figure S2. Gene ontology (GO) and pathway analysis were performed using 
MirTarBase [21]. All data were presented as means \pm SD. Graph Pad Prism (v7.0), $t$ tests, one-way ANOVA with post-hoc Tukey-Kramer HSD (cytokine analysis) and Spearman correlations were used along with Partek Genomics Suite 6.6 for paired sample $t$ test to determine differential microRNA expression.

\section{Results}

\section{Patient demographics}

A total of 16 healthy adult subjects (non-smokers) were enrolled in this study. The median age of the cohort was $27.94( \pm 4.28)$ years. Gender distribution was comprised of seven males and nine females.

\section{Divergent inflammatory response in alveolar}

\section{macrophages from alternate lobes of the lung with} oxygen stress

Primary alveolar macrophages from the right upper lobe (RUL) and the right lower lobe (RLL) of the lung were obtained from healthy volunteers via bronchoalveolar lavage, equilibrated overnight in vitro (normoxic) and then subject to continued normoxic $\left(21 \% \mathrm{O}_{2}\right)$ or hypoxic conditions $\left(5 \% \mathrm{O}_{2}\right)$ for $1 \mathrm{~h}$. Early response cytokines IL- 8 and TNF- $\alpha$ were measured by ELISA from conditioned media. A significant increase in IL- 8 and TNF- $\alpha$ secretion was detected from both upper lobe and lower lobe AM under oxygen stress (Fig. 1). Fold changes in cytokine secretion (up to 20x) were observed after just 60 minutes of in vitro hypoxia (5\% Oxygen exposure) from upper lobe AM (IL8: $31 \mathrm{pg} / \mathrm{ml}$ to $2295 \mathrm{pg} / \mathrm{ml}$ ) (ANOVA $P<0.0001)$, (TNFo: $34-2657 \mathrm{pg} / \mathrm{ml})(P<0.0001)$ and lower lobe AM (IL8: $31 \mathrm{pg} / \mathrm{ml}$ to $830 \mathrm{pg} / \mathrm{ml} P=0.004$ ) (TNFo: $35-1031 \mathrm{pg} / \mathrm{ml})(P=0.0051)$. Of particular note, illustrating a differential zonal response, lower lobe alveolar macrophages did not respond as prominently to hypoxia as did their upper lobe counterpart, consistent for both cytokines (IL8: Upper $2295 \mathrm{pg} / \mathrm{ml}$ vs. Lower $830 \mathrm{pg} / \mathrm{ml}(P<0.0001)$ and TNF $\alpha$ : Upper $2657 \mathrm{pg} / \mathrm{ml}$ vs Lower $1031 \mathrm{pg} / \mathrm{ml})(P<0.0001)$.

\section{Hypoxia influences microRNA expression in alveolar macrophages}

Regional changes in microRNA profiles and content were assayed in AM under in vitro normoxic $\left(21 \% \mathrm{O}_{2}\right)$ or hypoxic oxygen stress conditions $\left(5 \% \mathrm{O}_{2}-24 \mathrm{~h}\right)$. Of the 800 different microRNAs measureable with the NanoString nCounter miR assay platform, 73 microRNAs were detectable in AM samples. The top 10 highest expressed microRNAs were nearly identical in alveolar macrophages under all experimental conditions examined (Table 1).

This group of most highly expressed microRNAs from healthy lung macrophages include: three let-family members (let-7a-5p, let-7b-5p, and let-7d-5p), miR-23a,

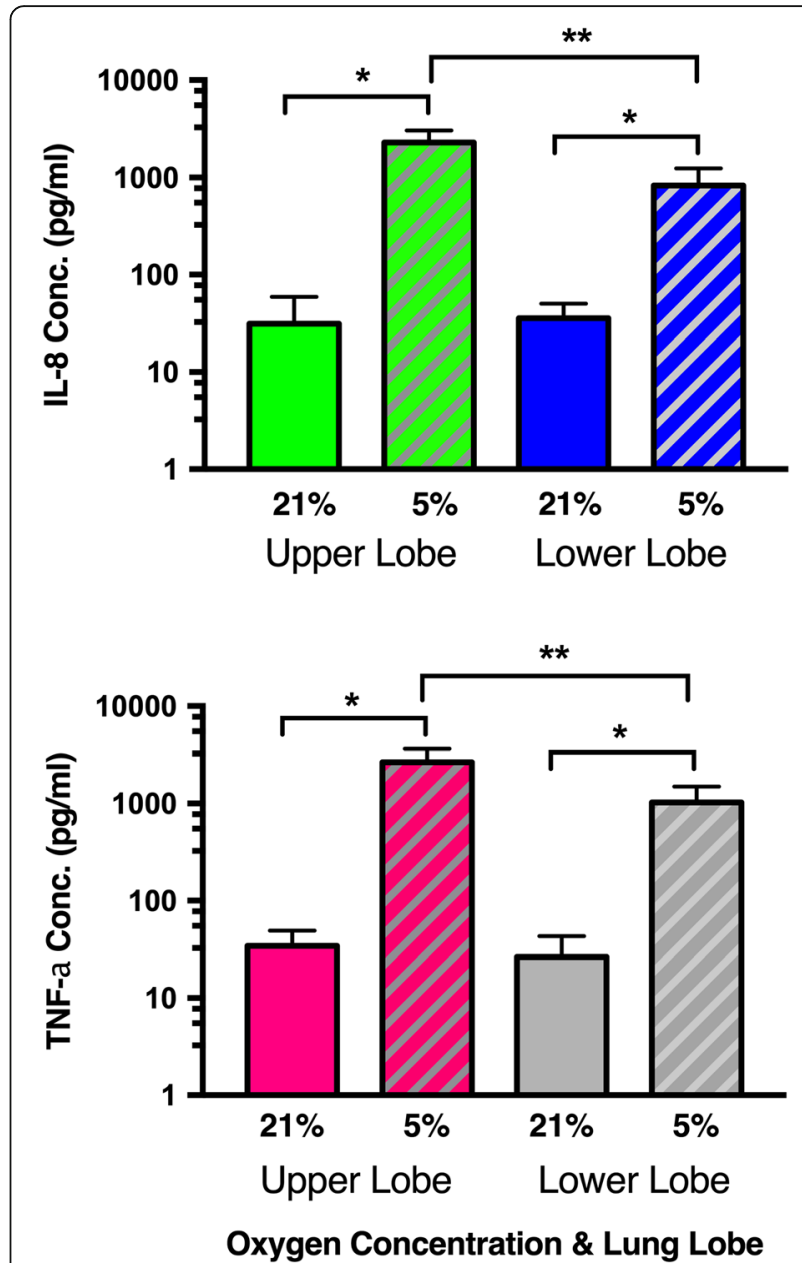

Fig. 1 Secretion of inflammatory cytokines by regional alveolar macrophages in response to hypoxia. Alveolar macrophages obtained via bronchoalveolar lavage (right upper and lower lobes) were subject to in vitro hypoxic conditions $\left(5 \% \mathrm{O}_{2}\right.$ for $\left.60 \mathrm{~min}\right)$ in an in vivo $_{2} 300$ hypoxic chamber (Baker Ruskinn). Cytokines IL-8 and TNF-a $(\mathrm{pg} / \mathrm{ml})$ were measured by ELISA from conditioned media. Basal levels of cytokines secreted from AM are $<35 \mathrm{pg} / \mathrm{ml}$ in $21 \% \mathrm{O}_{2}$, however a significant increase in cytokine secretion occurs when macrophages are oxygen stressed at 5\% $\mathrm{O}_{2}$ (ANOVA Tukey-Kramer HSD $P<0.0001)$. *Hypoxia induced increase in cytokine levels increase in both lobes $(P<0.0001)$. ${ }^{*}$ Cytokine secretion was quantitatively higher from upper lobe AM (IL-8: upper 2294.6 (+731.8) vs. lower 830.4 (+415.8) $\mathrm{pg} / \mathrm{ml})(P<0.0001)$. (TNF-a: upper 2656.9 (+986.8) vs. lower 1031.1 $(+464.1) \mathrm{pg} / \mathrm{ml})(P<0.0001)$. Results from three independent experiments are reported as the means $\pm \mathrm{SD}$

miR-223, miR-4454, and miR-155. Next, comparative analysis was done amongst microRNA profiles from the varying in vitro experimental conditions. First, we compared miRNA profiles from upper vs. lower AM under normoxic conditions. Log2 fold changes along with $P$ values are shown in Table 2 .

Three microRNAs were significantly different between regional macrophages miR-4443 $(P=0.003)$, miR-30e-5p $(P=0.018)$ and miR-4454 $(P=0.032)$. Next, miRNA 
Table 1 Top 10 microRNAs in alveolar macrophages (AM)

\begin{tabular}{lll}
\hline Gene name & Mean miRNA read counts & SD \\
\hline hsa-let-7a-5p & 5113 & 1983 \\
hsa-miR-23a-3p & 3162 & 1354 \\
hsa-let-7b-5p & 2964 & 1133 \\
hsa-miR-223-3p & 2802 & 1105 \\
hsa-miR-191-5p & 2785 & 1348 \\
hsa-miR-342-3p & 1929 & 733 \\
hsa-miR-4454 & 1090 & 1073 \\
hsa-miR-21 & 962 & 440 \\
hsa-miR-155 & 624 & 250 \\
hsa-let-7d-5p & 583 & 328
\end{tabular}

anput: 100 ngs total RNA

profiles from AM within each lobe were analyzed comparing normoxia to hypoxia. In the upper lobe, miR664a-3p $(P=0.005)$ was the lone microRNA identified as significantly changed upon $24 \mathrm{~h}$ exposure to $5 \% \mathrm{O}_{2}$. However, in the lower lobe, four microRNAs were significantly altered including: miR-22-3p $(P=0.007)$, miR30e-5p $(P=0.015)$, miR-4454 $(P=0.019)$, and miR-93-5p $(P=0.036)$.

\section{MicroRNA profiling of BAL Fluid}

Lung fluid from both the right upper lobe and the right lower lobe was obtained by performing bronchoalveolar lavage on 16 healthy subjects. MicroRNA was isolated, purified and quantitated. Prior to NanoString miRNA assay, BAL fluid was evaluated for presence of exosomes

Table 2 Differentially expressed MicroRNAs comparing upper lung and lower lung AM

\begin{tabular}{llll}
\hline microRNA ID & Expression change & $\log F C$ & $P$ value \\
\hline $\begin{array}{c}\text { Normoxia } \\
\text { Upper vs. lower }{ }^{\mathrm{a}}\end{array}$ & & & \\
hsa-miR-30e-5p & Increase & 0.313 & 0.018 \\
hsa-miR-4454 & Decrease & -0.986 & 0.032 \\
hsa-miR-4443 & Decrease & -0.757 & 0.003 \\
Hypoxia & & & \\
Upper lobe MO ${ }^{\text {b }}$ & & & \\
hsa-miR-664a-3p & Decrease & -0.498 & 0.005 \\
Lower lobe MO $^{\text {b }}$ & & & \\
hsa-miR-30e-5p & Increase & 0.412 & 0.015 \\
hsa-miR-93-5p & Increase & 0.306 & 0.036 \\
hsa-miR-4454 & Decrease & -1.057 & 0.019 \\
hsa-miR-22-3p & Decrease & -0.540 & 0.007 \\
\hline
\end{tabular}

microRNA ID = official microRNA name according to miRBASE v21; $\log F C=\log 2$ fold change in macrophages from upper lobe vs. lower lobe

$P$ value $=$ the unadjusted $P$ value from the statistical test (paired analysis)

a Upper lobe AM vs. lower lobe AM

${ }^{\mathrm{b}} 21 \% \mathrm{O}_{2}$ vs. $5 \% \mathrm{O}_{2}$ /microvesicles by negative stain electron microscopy (Fig. 2a, b). Exosomes /microvesicle size range was $50-$ $600 \mathrm{~nm}$. Of the 800 different microRNAs measureable with the NanoString nCounter platform, 35 microRNAs were detectable in BAL samples. Table 3 lists the four microRNAs with significant differences in expression levels comparing upper lobe and lower lobe BAL, plus $\log 2$ fold change and $P<0.05$, respectively.

Comparison of the changes from each BAL sample $(n=16)$ lower lobe vs. upper lobe $\log 2$ normalized expression of the two miRNAs with largest fold change are shown in Fig. 2 c, d. MiR-451a demonstrates reduced expression in upper compared to lower lobe BAL, while miR-663a shows higher expression. Additionally, the overall distribution of microRNA expression differences (log2-fold change) comparing upper to lower lobe BAL samples is presented in Fig. 2e. Droplet digital PCR via Taqman assay was used for independent validation of hsamiR-451a expression levels. Correlation plot for hsa-miR451a comparing platforms NanoString versus ddPCR reads is shown in Fig. $2 \mathrm{f}(r h o=0.7038, P=0.002)$.

\section{Gene ontology (GO) and pathway analysis}

Gene ontology was assessed using miRTarBase [21]. We performed in silico analysis of microRNAs identified differential expressed in BAL fluid to specifically identify potential gene targets of miR-451a and miR-663a. MicroRNA gene set enrichment networks (GSEN) identify 23 overall target genes for miR-451a, 10 of these with strong experimental evidence, including $M I F$, MMP-2, MMP-9 and AKT1 (Fig. 3a). Additionally, GSEN identified 105 possible miR-663a target genes, eleven of these with strong experimental evidence of interaction, including $C E B P \beta$ and TGF 1 (Fig. 3b). The top 10 experimentally validated gene targets of hsa-miR451a and hsa-miR-663a along with the GO Biological Process key words for each are shown in Table 4.

\section{Discussion}

Numerous pulmonary diseases occur with upper lobe predominance (ULP) including cystic fibrosis, smokingrelated COPD, tuberculosis, and sarcoidosis [1]; however, no specific initiating cellular mechanisms driving these pathologies have been defined. The current dogma explaining ULP lung diseases is that gravity induces regional differences in perfusion-ventilation ratio, partial pressure of oxygen and lymphatic flow within the lung $[1,2]$ leading to regional differences in the host-topathogen response-upper lobe versus lower lobe in the lung. One potentially important difference comparing upper lobe vs. lower lobe is the variance in oxygen concentration between the regions, which equates to a decreasing hypoxic gradient from the apex $\left(21 \% \mathrm{O}_{2}\right)$ to the base $\left(10 \% \mathrm{O}_{2}\right)$ of the lung. Hypoxia has been shown to 
a

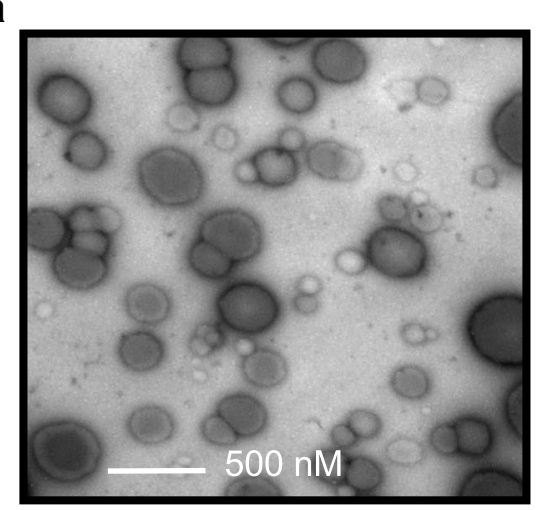

C

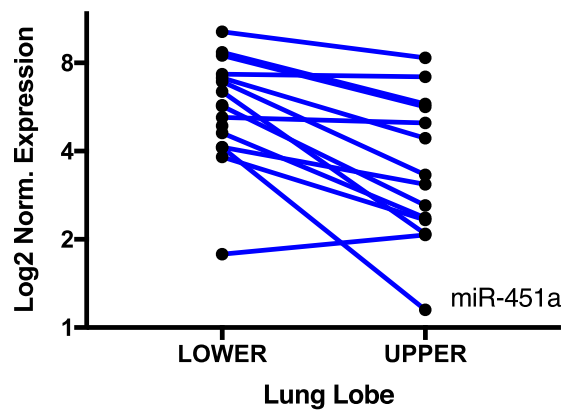

e

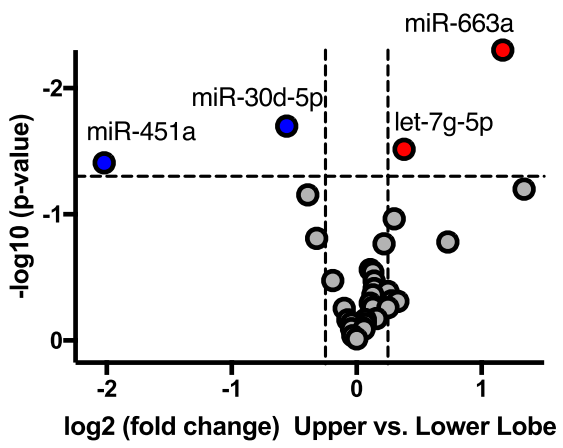

b

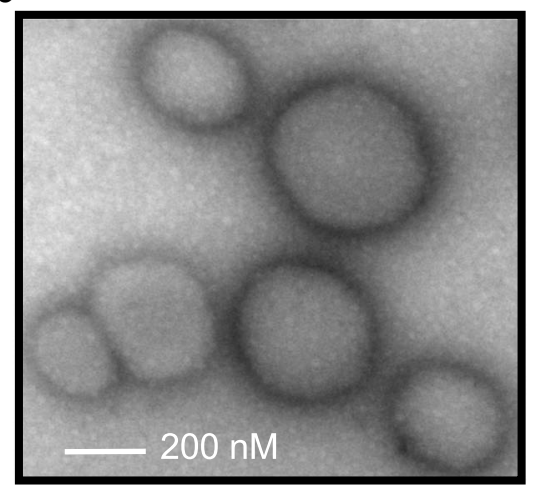

d

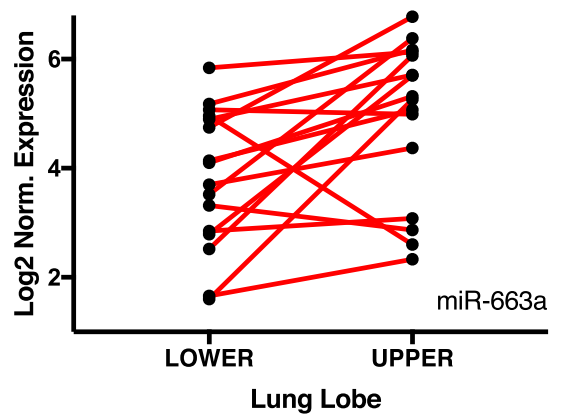

f

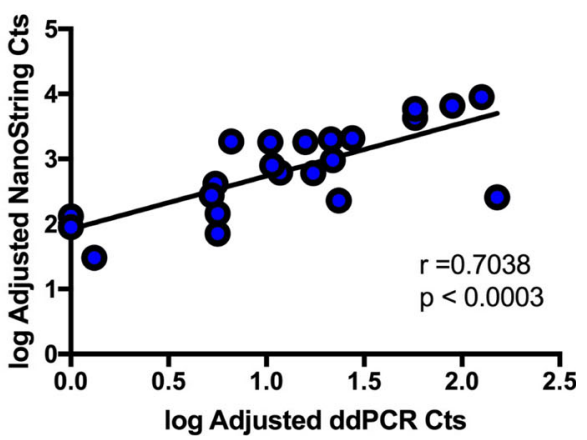

Fig. 2 Divergent microRNA expression from isolated microvesicles/exosomes of BAL Fluid - Right Upper Lobe (RUL) vs. Right Lower Lobe (RLL). Microvesicles/exosomes in BAL fluid visualized via negative stain electron microscopy with a JEOL TEM1010 transmission electron microscope (a + b) (scale bar in nanometers). Individual microRNAs in BAL with highest log2 fold change RUL vs. RLL BAL fluid: miR-451a (c) $(P=0.039)$ \& miR663a (d) $(P=0.005)$. Each line represents directional change in individual samples (total $n=16)$. Volcano plot of microRNA expression differences (log2 fold change) from BAL samples (e). Scatterplot of ddPCR with Taqman assays and NanoString counts ofmicroRNA-451a, coefficient reported from Spearman correlation $(\mathbf{f})(r h o=0.7038)(P<0.003)$

Table 3 Differentially expressed MicroRNAs in upper lung vs. lower lung BAL fluid

\begin{tabular}{llll}
\hline Expression change & & & \\
\hline microRNA ID & UL vs. LL & logFC & $P$ value \\
\hline hsa-miR-663a & Increase & 1.17 & 0.005 \\
hsa-let-7 g-5p & Increase & 0.38 & 0.031 \\
hsa-miR-451a & Decrease & -2.02 & 0.039 \\
hsa-miR-30d-5p & Decrease & -0.56 & 0.020 \\
\hline
\end{tabular}

microRNA ID = official microRNA name according to miRBASE v21; $\log F C=\log 2$ fold change in BAL from upper lobe vs. lower lobe; $P$ value $=$ the unadjusted $P$ value from the statistical test (paired analysis) modulate multiple aspects of cell biology [5, 18]. Our goal was to investigate possible cellular mechanisms influencing regional differences in pulmonary cellular response associated with hypoxia. We hypothesized that the basal hypoxic gradient of the healthy lung may preprogram regional pulmonary cells to respond dissimilarly, conditional on the decreasing oxygen gradient concentrations. The reasoning behind gaining a better understanding of the regional pulmonary response is that this differential response could ultimately lead to differing innate immune responses to invading pathogens, 


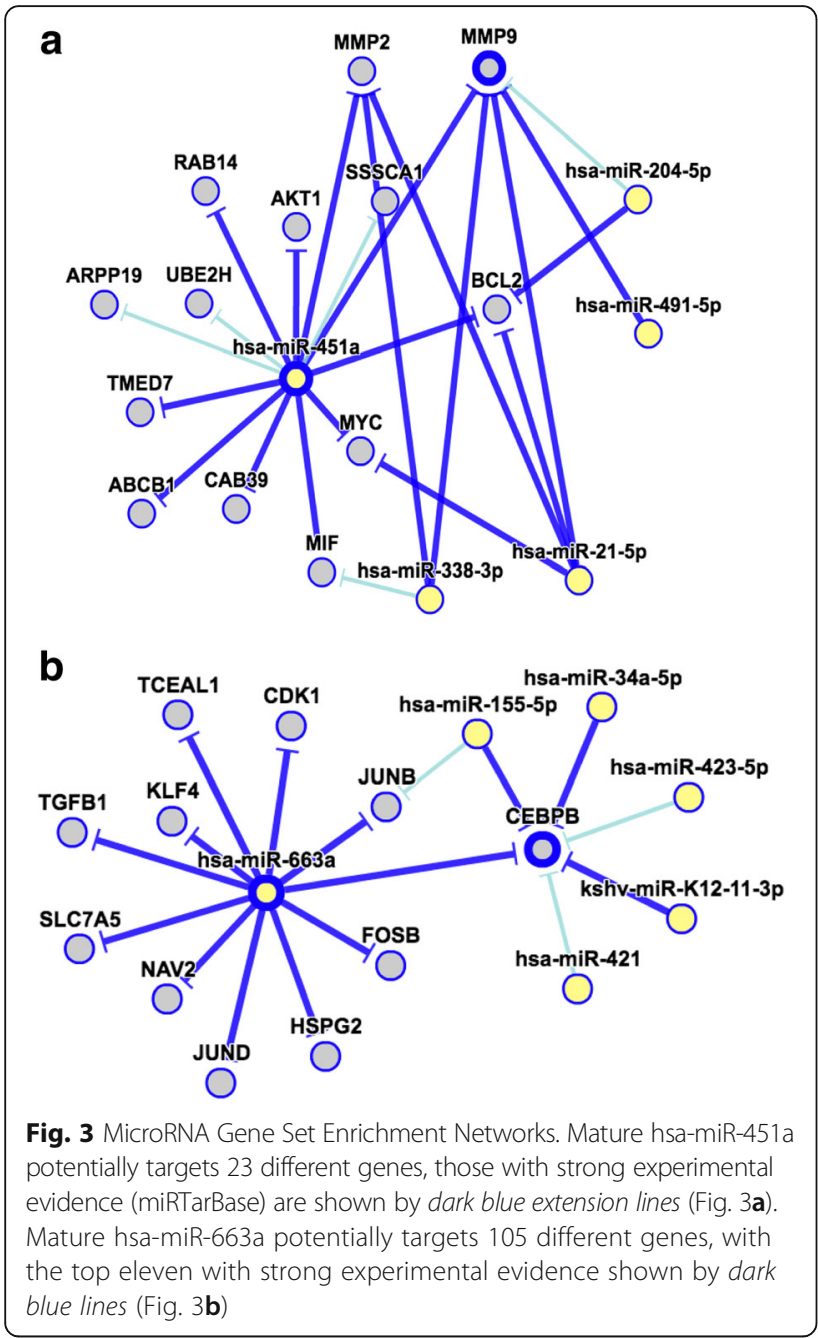

pathogen colonization and/or disease progression. Studies presented here were conducted in the healthy lung to lay the groundwork for subsequent studies and a more precise interpretation of effects in the diseased lung. Additionally, we chose to focus primarily on microRNA, as these small non-coding RNA molecules are likely involved in a broad scope of transcriptional regulation and are increasingly being recognized as important modulators in respiratory disease $[11,12]$.

A number of recent studies have emerged in lung cancer, pulmonary fibrosis, chronic obstructive pulmonary disease (COPD), asthma, and cystic fibrosis, implicating microRNAs in the molecular pathogenesis of these lung diseases [22-24]. Additionally, hypoxia-dependent covariant microRNA biogenesis and secretion has also been demonstrated [25], where it was determined that the exosomal microRNA secretome is altered in response to hypoxia.

This study, as far as we know, is the first to report microRNA profiling from both regional alveolar macrophages and regional BAL fluid. Our approach is unique in that it provides a direct comparison of the epigenetic molecular signature of the upper lobe vs. the lower lobe from each of these primary human bio-specimen sources.

Our initial in vitro experiments demonstrating a hypoxia-associated early response, and regional differential IL- 8 and TNF- $\alpha$ secretion from AM, proved interesting, demonstrating hypoxia-associated effects in upper lobe vs. lower lobe AM. This led us to wonder about the broader extent of zonal AM gene modulation with oxygen stress. We focused the remainder of our effort at the level of epigenetic transcriptional regulation. To examine more closely the potential cellular mechanisms involved here, we chose to investigate transcriptional regulation via a high-throughput microRNA profiling platform. In addition, we chose a 24-h hypoxia, as this more closely simulates the in vivo $\mathrm{O}_{2}$ steady state of AM in lung.

As a first approach, we examined the microRNA content of AM and identified a number of microRNAs that play roles related to innate immunity, inflammation or extracellular matrix remodeling including: miR-93-5p, miR-22-3p and miR-4454. Potential targets identified via miRTarBase [21] of miR-93-5p include PTEN [26] and Il-8 [27], of miRNA-22-5p include PPARA and BMP7 [28] and HMGB1 [29], and of miR-4454 include TRAM2 and CCR4. MicroRNA regulation of cellular processes is a complicated issue as each specific miRNA can have a multitude of mRNA targets [13]. The one overlap we noted between our protein data and microRNA data was the observation of the increase of miR-93-5p expression seen in lower lobe AM is consistent with our observed decreased of IL- 8 secretion by AM in vitro.

Next, we wanted to determine if we could measure a regional difference in microRNA from the exosomes/ microvesicles secreted or shed into the BAL fluid. Only two studies to date have examined microRNA in human BAL fluid [30,31] and neither addressed the regional distribution of microRNAs within the lungs. Interestingly, miRNA content of regional BAL fluid in this study shows divergence of several microRNAs that could prove to be of importance in inflammation within the lung, namely miR-451a and miR-663. miRTarBase gene set enrichment network analysis has identified several experimentally validated targets of miR-451a that are certainly intriguing in the context of innate immunity including: $M I F$ [32-34] and $M M P-2$ and MMP-9 [35, 36]. MIF could potentially alter macrophage movement regionally and MMP-2 and MMP-9 may alter extracellular matrix structural integrity, ultimately influencing altered fibrosis regionally. It's not readily apparent how the differential levels of miR451a, or other microRNAs we identified effect their potential targets in vivo. Likewise, it has also not been established the exact validated targets ultimately effected in this setting. 
Table 4 Validated gene targets of hsa-miR-451a \& hsa-miR-663a and GO Biological Processes Key Words

\begin{tabular}{|c|c|c|}
\hline Gene ID & Name & GO Biological Process Key Words \\
\hline \multicolumn{3}{|c|}{ miR-451a gene targets } \\
\hline MIF & (Macrophage migration inhibitory factor) & Immunity, inflammatory response, innate immunity \\
\hline $\mathrm{BCL} 2$ & (B cell lymphoma 2) & apoptosis \\
\hline CAB39 & (calcium binding protein 39) & cell cycle arrest \\
\hline ABCB1 & (Multidrug resistance protein 1) & transport \\
\hline AKT1 & (RAC-alpha serine/threonine-protein kinase) & apoptosis \\
\hline MMP-2 & (Matrix metalloproteinase 2) & angiogenesis, collagen degradation \\
\hline MMP-9 & (Matrix metalloproteinase 9) & collagen degradation \\
\hline RAB14 & (RAS-related protein Rab-14) & protein transport, transport \\
\hline RAB5A & (RAS-related protein Rab-5A) & endocytosis, phagocytosis \\
\hline IL6R & (IL6 Receptor) & cytokine mediated signaling pathway \\
\hline \multicolumn{3}{|c|}{ miR-663a gene targets } \\
\hline JUNB & (jun B proto-oncogene) & transcription, transcriptional regulation \\
\hline JUND & (jun D proto-oncogene) & transcription, transcriptional regulation \\
\hline KLF4 & (Kruppel-like factor 4) & transcription, transcriptional regulation \\
\hline FOSB & (FBJ murine osteosarcoma viral oncogene) & transcription regulation (AP1) \\
\hline SLC7A5 & (solute carrier family 7, member 5 ) & amino acid transport (lung) \\
\hline NAV2 & (neuron navigator 2) & helicase, hydrolase \\
\hline CEBPB & (CCAAT/enhancer binding protein, beta) & transcription, transcriptional regulation \\
\hline HSPG2 & (heparin sulfate proteoglycan 2-perecan) & angiogenesis, (basement membrane) \\
\hline CDK1 & (cyclin-dependent kinase 1) & apoptosis, cell cycle \& division, mitosis \\
\hline TGFB & (transforming growth factor beta) & inflammatory response \\
\hline
\end{tabular}

It is also worth noting at this point that the zonally divergent microRNAs detected in BAL fluid did not overlap with the hypoxia-divergent microRNAs detected within macrophages. This may simply indicate that the cell source(s) of miRNAs in BAL fluid is a cell-type other than the alveolar macrophage, consistent with numerous studies having demonstrated cell-type-specific microRNA expression [37-39].

An emerging area of interest in eukaryotic biology is the subject of trans-kingdom cross-talk, whereby small molecules produced by the host influence a symbiont or pathogen. MicroRNAs are at the forefront of these types of studies. It is possible that some of the microRNAs detected here may be functioning in a host-pathogen trans-kingdom cross-talk role. For instance, a BLAST of the hsa-miR-451a mature sequence on the fungal and oomycete genomics database: FungiDB, reveals 100\% sequence homology to gene Afu5g11540 - localized to the mitochondrial ribosome of Aspergillis fumigatus. Aspergillis fumigatus is one of the main fungal species found in cystic fibrosis airways [40]. If hsa-miR-451a does influence Aspergillis fumigatus growth, it is easy to see how differing levels of this non-coding RNA regionally in the human lung may alter the hostpathogen response. Future studies to address this topic in the context of a lung disease such as cystic fibrosis could prove very useful in better understanding the host-pathogen relationship.

Our current study has a number of strengths. The availability and analysis of regional lung macrophages and BAL fluid microRNA content makes this pulmonary study unique. Additionally, the identification of divergent zonal microRNA profiles coupled with the use of in silico analysis by miRTarBase allows for development of new hypotheses linking experimentally validated microRNA targets and innate immunity pathways.

This work, however, also has a number of limitations. Firstly, this is a descriptive study and as such does not address the specific intracellular or intercellular regulatory mechanisms of microRNA within the lung. Additionally, identifying the specific cell source(s) of the microRNA in BAL fluid was beyond the scope of this study.

\section{Conclusions}

In summary, we present evidence that the microRNA complement of the upper lobe of the lung is different than the lower lobe and suggest that hypoxia within the lung may at least in part be responsible for these molecular differences. We have demonstrated that hypoxia can drive alterations in microRNA content in alveolar 
macrophages potentially influencing the inflammatory response. Additionally, basal microRNA content of upper lobe BAL fluid is different than lower lobe BAL fluid, indicating an association with the natural hypoxic gradient that exists from the apex to the base of the lung.

Ultimately, it remains to be determined if the specific microRNAs identified in this study, miR-93, miR-4454, miR-451a or miR-663a, function intracellularly, in a paracrine or endocrine manner in cell-to-cell communication, or are involved in trans-kingdom crosstalk with potential pathogens, or some combination of these processes.

\section{Additional files}

Additional file 1: Online supplemental methods. (DOCX $97 \mathrm{~kb}$ )

Additional file 2: Figure S1. Exosomes/microvesiclescontaining microRNAs were isolated from Bronchoalveolar Lavage (BAL) Fluid. Agilent Bio-Analyzer was used to quantitate microRNA recovery. $1 \mu \mathrm{l}$ per sample run on BioAnalyzer 2100 with the Small RNA Chip kit. MlcroRNA seen at 20-40 nt, additional peaks in electropherogram represent tRNA and other small RNAs. (RLL: right lower lobe; RUL: right upper lobe). (TIFF 563 kb)

Additional file 3: Figure S2. Correlation of NanoString nCounter microRNA assay technical replicates. Technical replicates of alveolar macrophage total RNA were run on the NanoString nCounter microRNA assay. Strong correlation was seen between replicates (rho $=0.987 P<0.0001$ ). (PDF $67 \mathrm{~kb}$ )

\section{Abbreviations}

AM: Alveolar macrophage; BAL: Bronchoavelolar lavage; CF: Cystic fibrosis; ddPCR: Droplet digital polymerase chain reaction; GO: Gene ontology; GSEN: Gene set enrichment network; miRNA: MicroRNA

\section{Acknowledgements}

The authors would like to thank members of the Department of Pulmonary and Critical Care Medicine at Dartmouth Hitchcock Medical Center, for coordination of collection and distribution of clinical samples used in this work. Clinical Coordinators include John Dessaint, Dana Dorman, Brian Aldrich, Molly Williams, Barbara Rogers, Nicola Felicitti, and Lynn Freeman.

\section{Funding}

This work is supported by the NIH R01HL122372 grant, received August of 2015

\section{Availability of data and materials}

All data used in this study is available upon request from the corresponding author.

\section{Authors' contributions}

$\mathrm{AA}$ and DAA contributed to the conception and design. DAA, ABN, HFH, and $\mathrm{LH}$ contributed to the acquisition of data. DAA, CL, CR, and $\mathrm{AA}$ contributed to the analysis and interpretation. DAA, CL, ABN, HFH, and $\mathrm{AA}$ contributed to the drafting of the manuscript for important intellectual content. All authors contributed toward critical review and approval of the manuscript and are accountable for all aspects of the work published.

\section{Competing interests}

The authors declare that they have no competing interests.

\section{Consent for publication}

Not applicable.

\section{Ethics approval and consent to participate}

This study was approved by the Committee for the Protection of Human Subjects at the Geisel School of Medicine at Dartmouth (\#22781). All study volunteers provided written informed consent for participation.

\section{Publisher's Note}

Springer Nature remains neutral with regard to jurisdictional claims in published maps and institutional affiliations.

\section{Author details}

${ }^{1}$ Pulmonary and Critical Care Medicine, Dartmouth-Hitchcock Medical Center, Lebanon, NH, USA. ${ }^{2}$ Geisel School of Medicine at Dartmouth, Hanover, NH, USA. ${ }^{3}$ Department of Environmental Medicine and Public Health, Icahn School of Medicine at Mount Sinai, New York, NY, USA. ${ }^{4}$ Department of Environmental Health, Rollins School of Public Health, Emory University, Atlanta, GA, USA.

Received: 11 April 2017 Accepted: 16 May 2017

Published online: 30 May 2017

\section{References}

1. Nemec SF, Bankier AA, Eisenberg RL. Upper lobe-predominant diseases of the lung. AJR Am J Roentgenol. 2013;200(3):W222-37.

2. West JB. Regional differences in gas exchange in the lung of erect man. J Appl Physiol. 1962;17:893-8.

3. Carreau A, El Hafny-Rahbi B, Matejuk A, Grillon C, Kieda C. Why is the partial oxygen pressure of human tissues a crucial parameter? Small molecules and hypoxia. J Cell Mol Med. 2011;15(6):1239-53.

4. Egners A, Erdem M, Cramer T. The Response of Macrophages and Neutrophils to Hypoxia in the Context of Cancer and Other Inflammatory Diseases. Mediators Inflamm. 2016:2016:2053646.

5. Leblond MM, Gerault AN, Corroyer-Dulmont A, MacKenzie ET, Petit E, Bernaudin M, Valable S. Hypoxia induces macrophage polarization and reeducation toward an M2 phenotype in U87 and U251 glioblastoma models. Oncoimmunology. 2016:5(1):e1056442.

6. Stafford $J L$, Neumann NF, Belosevic M. Macrophage-mediated innate host defense against protozoan parasites. Crit Rev Microbiol. 2002;28(3):187-248.

7. Chuquimia OD, Petursdottir DH, Periolo N, Fernandez C. Alveolar epithelial cells are critical in protection of the respiratory tract by secretion of factors able to modulate the activity of pulmonary macrophages and directly control bacterial growth. Infect Immun. 2013;81(1):381-9.

8. Wu C, Morris JR. Genes, genetics, and epigenetics: a correspondence. Science. 2001;293(5532):1103-5.

9. Maccani MA, Padbury JF, Lester BM, Knopik VS, Marsit CJ. Placental miRNA expression profiles are associated with measures of infant neurobehavioral outcomes. Pediatr Res. 2013:74(3):272-8.

10. Vencken SF, Greene CM, McKiernan PJ. Non-coding RNA as lung disease biomarkers. Thorax. 2015;70(5):501-3.

11. Maltby S, Plank M, Tay HL, Collison A, Foster PS. Targeting MicroRNA Function in Respiratory Diseases: Mini-Review. Front Physiol. 2016;7:21.

12. Rupani H, Martinez-Nunez RT, Dennison P, Lau LC, Jayasekera N, Havelock T, Francisco-Garcia AS, Grainge C, Howarth PH, Sanchez-Elsner T. Toll-like Receptor 7 Is Reduced in Severe Asthma and Linked to an Altered MicroRNA Profile. Am J Respir Crit Care Med. 2016;194(1):26-37.

13. Chen $X$, Liang $H$, Zhang J, Zen $K$, Zhang CY. Secreted microRNAs: a new form of intercellular communication. Trends Cell Biol. 2012;22(3):125-32.

14. Belov L, Matic K, Hallal S, Best OG, Mulligan SP, Christopherson RI. Extensive surface protein profiles of extracellular vesicles from cancer cells may provide diagnostic signatures from blood samples. J Extracell Vesicles. 2016;5:25355

15. Kozomara A, Griffiths-Jones S. miRBase: annotating high confidence microRNAs using deep sequencing data. Nucleic Acids Res. 2014; 42(Database issue):D68-73.

16. Mehta A, Baltimore D. MicroRNAs as regulatory elements in immune system logic. Nat Rev Immunol. 2016;16(5):279-94.

17. Knip M, Constantin ME, Thordal-Christensen H. Trans-kingdom cross-talk: small RNAs on the move. PLoS Genet. 2014;10(9):e1004602.

18. Nallamshetty S, Chan SY, Loscalzo J. Hypoxia: a master regulator of microRNA biogenesis and activity. Free Radic Biol Med. 2013;64:20-30. 
19. Naqvi AR, Zhong S, Dang H, Fordham JB, Nares S, Khan A. Expression Profiling of LPS Responsive miRNA in Primary Human Macrophages. J Microb Biochem Technol. 2016;8(2):136-43.

20. Seidler D, Griffin M, Nymon A, Koeppen K, Ashare A. Throat Swabs and Sputum Culture as Predictors of P. aeruginosa or S. aureus Lung Colonization in Adult Cystic Fibrosis Patients. PLoS One. 2016;11(10):e0164232.

21. Chou CH, Chang NW, Shrestha S, Hsu SD, Lin YL, Lee WH, Yang CD, Hong HC, Wei TY, Tu SJ, et al. miRTarBase 2016: updates to the experimentally validated miRNA-target interactions database. Nucleic Acids Res. 2016;44(D1):D239-47.

22. Brown $D$, Rahman M, Nana-Sinkam SP. MicroRNAs in respiratory disease. A clinician's overview. Ann Am Thorac Soc. 2014;1 1(8):1277-85.

23. Conickx G, Mestdagh P, Avila Cobos F, Verhamme FM, Maes T, Vanaudenaerde BM, Seys LJ, Lahousse L, Kim RY, Hsu AC, Wark PA, Hansbro PM, Joos GF, Vandesompele J, Bracke KR, Brusselle GG. MicroRNA Profiling Reveals a Role for MicroRNA-218-5p in the Pathogenesis of Chronic Obstructive Pulmonary Disease. Am J Respir Crit Care Med. 2017;195(1):43-56. doi:10.1164/rccm. 201506-11820C

24. McKiernan PJ, Greene CM. MicroRNA Dysregulation in Cystic Fibrosis. Mediators Inflamm. 2015;2015:529642.

25. Gray WD, French KM, Ghosh-Choudhary S, Maxwell JT, Brown ME, Platt MO, Searles CD, Davis ME. Identification of therapeutic covariant microRNA clusters in hypoxia-treated cardiac progenitor cell exosomes using systems biology. Circ Res. 2015;116(2):255-63.

26. Fu X, Tian J, Zhang L, Chen Y, Hao Q. Involvement of microRNA-93, a new regulator of PTEN/Akt signaling pathway, in regulation of chemotherapeutic drug cisplatin chemosensitivity in ovarian cancer cells. FEBS Lett. 2012;586(9):1279-86.

27. Fabbri E, Borgatti M, Montagner G, Bianchi N, Finotti A, Lampronti I, Bezzerri V, Dechecchi MC, Cabrini G, Gambari R. Expression of microRNA-93 and Interleukin-8 during Pseudomonas aeruginosa-mediated induction of proinflammatory responses. Am J Respir Cell Mol Biol. 2014;50(6):1144-55.

28. Iliopoulos D, Malizos KN, Oikonomou P, Tsezou A. Integrative microRNA and proteomic approaches identify novel osteoarthritis genes and their collaborative metabolic and inflammatory networks. PLoS One. 2008;3(11):e3740.

29. Lee SA, Kwak MS, Kim S, Shin JS. The role of high mobility group box 1 in innate immunity. Yonsei Med J. 2014;55(5):1165-76.

30. Brock M, Rechsteiner T, Kohler M, Franzen D, Huber LC. Kinetics of microRNA Expression in Bronchoalveolar Lavage Fluid Samples. Lung. 2015;193(3):381-5.

31. Brown JN, Brewer HM, Nicora CD, Weitz KK, Morris MJ, Skabelund AJ, Adkins $\mathrm{JN}$, Smith RD, Cho JH, Gelinas R. Protein and microRNA biomarkers from lavage, urine, and serum in military personnel evaluated for dyspnea. BMC Med Genomics. 2014;7:58.

32. Carli C, Metz CN, Al-Abed Y, Naccache PH, Akoum A. Up-regulation of cyclooxygenase-2 expression and prostaglandin E2 production in human endometriotic cells by macrophage migration inhibitory factor: involvement of novel kinase signaling pathways. Endocrinology. 2009;150(7):3128-37.

33. Graham A, Falcone T, Nothnick WB. The expression of microRNA-451 in human endometriotic lesions is inversely related to that of macrophage migration inhibitory factor (MIF) and regulates MIF expression and modulation of epithelial cell survival. Hum Reprod. 2015;30(3):642-52.

34. Yang Y, Degranpre P, Kharfi A, Akoum A. Identification of macrophage migration inhibitory factor as a potent endothelial cell growth-promoting agent released by ectopic human endometrial cells. J Clin Endocrinol Metab. 2000;85(12):4721-7.

35. Chakrabarti S, Patel KD. Matrix metalloproteinase-2 (MMP-2) and MMP-9 in pulmonary pathology. Exp Lung Res. 2005;31(6):599-621.

36. Cutroneo KR. How is Type I procollagen synthesis regulated at the gene level during tissue fibrosis. J Cell Biochem. 2003;90(1):1-5.

37. Oger F, Gheeraert C, Mogilenko D, Benomar Y, Molendi-Coste O, Bouchaert E, Caron S, Dombrowicz D, Pattou F, Duez H, et al. Cell-specific dysregulation of microRNA expression in obese white adipose tissue. J Clin Endocrinol Metab. 2014;99(8):2821-33.

38. Vrba L, Garbe JC, Stampfer MR, Futscher BW. Epigenetic regulation of normal human mammary cell type-specific miRNAs. Genome Res. 2011;21(12):2026-37.

39. Allantaz F, Cheng DT, Bergauer T, Ravindran P, Rossier MF, Ebeling M, Badi L, Reis B, Bitter H, D'Asaro M, et al. Expression profiling of human immune cell subsets identifies miRNA-mRNA regulatory relationships correlated with cell type specific expression. PLoS One. 2012;7(1):e29979.

40. Cowley AC, Thornton DJ, Denning DW, Horsley A. Aspergillosis and the role of mucins in cystic fibrosis. Pediatr Pulmonol. 2017;52(4):548-55.

\section{Submit your next manuscript to BioMed Central and we will help you at every step:}

- We accept pre-submission inquiries

- Our selector tool helps you to find the most relevant journal

- We provide round the clock customer support

- Convenient online submission

- Thorough peer review

- Inclusion in PubMed and all major indexing services

- Maximum visibility for your research

Submit your manuscript at www.biomedcentral.com/submit
C Biomed Central 